\title{
Flow Induced Vibration of Cantilever Tapered Pipes Transporting Fluid
}

\author{
MOHAMED GAITH \\ Mechanical Engineering Department \\ Al-Balqa' Applied University \\ P.O.Box 710709, Amman (11171) \\ JORDAN
}

\begin{abstract}
A cantilevered tapered slender pipe conveying an incompressible, inviscid fluid of one material is not a conserved system. For certain large fluid velocity, the pipe with uniform cross section would go unstable via flutter Hopf bifurcation. In this paper, the flow induced vibration for cantilever tapering pipe transporting a fluid is presented. Euler Bernoulli and Hamilton's theories are applied to develop the mathematical model which will be solved using well known Galerkan's procedure. The effect of smooth tapering of the circular cross sectional area, flow velocity and pipe to fluid mass fraction on the complex natural frequencies and stability will be investigated.
\end{abstract}

Abstract: - Complex frequencies, Dynamic stability, Tapered pipe.

Received: July 3, 2020. Revised: December 24, 2020. Accepted: January 12, 2021. Published: January 31, 2021.

\section{Introduction}

Due to its practical applications, cantilevered tubular pipes are common in many practical application such as chemical plants, hydraulic systems, and heat exchangers. Hence, several studies addressed the vibration and dynamics of such systems to reveal the impact of several factors on the behavior of vibrating pipe system such as conveying flow velocities, pipe material properties and boundary conditions [1]-[10]. Benjamin [1] used lagrangian approach and presented theoretical and experimental the vibration characteristics of piping system with free end. Gregory et al.[2] used different method to define the unstable regions for a cantilever system and validated the results experimentally with very good results. Paidoussis [15] considered the hanging and standing continuous cantilever pipes and investigated the stability maps for different parameters. Paidoussis et al.[16] considered the constant steady and variable flow velocity. They confirmed the flutter mode of instability for cantilever system, and flutter and bucking mode for simply supported systems. Ariaratnam et al.[17] used the method of averaging in studying the dynamics of pipe when the flow is pulsating. Johnson et al.[18] considered compressible flow for a cantilever tubular pipe using Newton's and
Muller's methods. Sugiyama et al.[19] revealed theoretically and experimentally the influence of mass lumped system on vibration stability of cantilever uniform pipe containing flow fluid. Vibration features of a pipe with functionally graded material containing a flow liquid was conducted analytically by Wang et al.[20]. In efforts to enhance the dynamic stability of pipe conveying air, Pisarski et al.[21] used electromagnetic tools. An increase of $50 \%$ of the critical fluid velocity is reached when they attached the actuators at the mid of the pipe span. Bai et al.[22] considered the dynamics of cantilever pipe with variable density and correlated to the system's stability. Wang et al.[23] addressed geometric and electromechanical coupling nonlinearity for a piezoelectric cantilevered pipe. They concluded that the added resistive piezoelectric damping decreases the flutter velocity. In most of the above studies, the pipe was assumed uniformed cross section along the span. Very few who considered the tapered pipes conveying fluid but with sudden change in pipe diameter [24],[25]. Pipes conveying fluid with variable cross section can be found, for instant, in many nuclear engineering like pipe expanding and nozzle. Gaith [26], [27] considered the non-uniform cross section of a simply supported pipe containing 
running fluid resting on Winkler viscoelastic layer. $\mathrm{He}$ presented the ranges of the buckling and coupled-mode flutter.

In this paper, the tapered slender cantilevered tubular pipe conveying constant steady flow velocity will be considered. The effect of tapering pipe and fluid pipe mass fraction on the natural frequencies and stability will be discussed.

\section{Formulation of the Problem}

Fig. 1 shows the pipe of finite length of $L$ along the axial coordinate $\mathrm{x}$-direction, with inlet diameter $\boldsymbol{b}$, outlet diameter $\boldsymbol{a}$, and pipe and fluid mass fraction $\frac{m_{p}(x)}{m_{f}(x)+m_{p}(x)}$ with constant inlet flow velocity $v_{o}$.

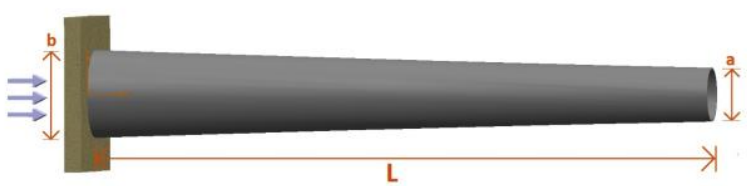

Fig. 1. Variable cross sectional Cantilever pipe containing flow fluid.

The pipe is with fixed-free supports and variable cross section is mathematically modeled by applying energy variation method [2]:

$E I \frac{\partial^{4} y}{\partial x^{4}}+\left(2 E \frac{d I}{d x}\right) \frac{\partial^{3} y}{\partial x^{3}}+\left(E \frac{d^{2} I}{d x^{2}}+m_{p} V^{2}\right) \frac{\partial^{2} y}{\partial x^{2}}+$

$2 m_{p} V \frac{\partial^{2} y}{\partial x \partial t}+\left(m_{p}+m_{f}\right) \frac{\partial^{2} y}{\partial t^{2}}=0$

$y(x, t)$ represents the vertical vibration of the piping system, $E I(x)$ is the variable flexural rigidity, $V(x)$ is the variable flow speed along the axial span with end conditions defined as:

$$
y(0, t)=\frac{\partial y(0, t)}{\partial x}=0 \text {, and } \frac{\partial^{2} y(L, t)}{\partial x^{2}}=\frac{\partial^{3} y(L, t)}{\partial x^{3}}=0
$$

The complete solution of the vertical displacement of the pipe system could possess a nondimensionalized

$Y(X, T)=W(X) e^{i \omega T}$, eq (1) becomes: $\frac{d^{4} W}{d X^{4}}+K_{1} \frac{d^{2} W}{d X^{3}}+K_{2} \frac{d^{2} W}{d X^{2}}+K_{3} \frac{d W}{d X}+K_{4} W=0$

with:

$K_{1}=\frac{8\left(\frac{b}{a}-1\right)}{\left(1+\left(\frac{b}{a}-1\right) X\right)}$

$K_{2}=\frac{12\left(\frac{b}{a}-1\right)^{2}}{\left(1+\left(\frac{b}{a}-1\right) X\right)^{2}}+U^{2} \frac{4\left(\frac{b}{a}\right)^{4}}{\pi\left(1+\left(\frac{b}{a}-1\right) X\right)^{6}}$

$K_{3}=2 \beta^{\frac{1}{2}} U\left(\frac{(2)\left(\frac{b}{a}\right)^{2}}{\sqrt{\pi}\left(1+\left(\frac{b}{a}-1\right) X\right)^{3}}\right) i \omega$

$K_{4}=-\omega^{2} \frac{4}{\pi\left(1+\left(\frac{b}{a}-1\right) X\right)^{4}}$

$B=\frac{1}{1+\beta^{*}\left(\frac{2 \bar{h}}{\left(1+\frac{b}{a}-1\right) X}\right)}, U=\left(\frac{\rho_{f} \pi}{E}\right)^{\frac{1}{2}} \frac{L v_{o}}{a^{2}}$

$W=\frac{w}{L} \quad, \quad X=\frac{x}{L}, \omega=\left(\frac{m_{f}+m_{p}}{E I}\right)^{\frac{1}{2}} L^{2} \Omega$

Where $\Omega$ is the complex circular frequency and $\beta^{*}=\frac{m_{p}(x)}{m_{f}(x)+m_{p}(x)} \quad$ is resembling mass fraction.

The fundamental natural frequency is obtained by applying fixed and free boundary conditions at both ends. The mode shape solution of the system can be calculated through the Galerkin's procedure assuming the mode function as [2] [26]:

$W_{i}(X)=\cos \left(\lambda_{i} X\right)-\cosh \left(\lambda_{i} X\right)$
$-\alpha\left[\sin \left(\lambda_{i} X\right)-\sinh \left(\lambda_{i} X\right)\right]$

Where:

$\alpha=\frac{\cos \left(\lambda_{i}\right)-\cosh \left(\lambda_{i}\right)}{\sin \left(\lambda_{i}\right)-\sinh \left(\lambda_{i}\right)}$

\section{Results and Discussion:}

In order to validate the model, the natural frequencies for are compared as shown in the Table 1 for the case $B=0.200, b / a=1.0$ at different velocities. Table 1 shows excellent comparison between the current model and the well-known 
results found in literature [2] for the first two complex natural frequencies. A finite element package is used to model the system in order to compare with current numerical results. For the case of $b / a=1.42, \quad B=0.223$, the first three natural frequencies and corresponding mode

Table 1. Comparison for first natural frequencies between current and literature values for $B=0.200, b / a=1.0$.

\begin{tabular}{|c|c|c|c|c|c|c|c|c|}
\hline & \multicolumn{2}{|c|}{$\operatorname{Re}\left(\omega_{1}\right)$} & \multicolumn{2}{c|}{$\operatorname{Im}\left(\omega_{1}\right)$} & \multicolumn{2}{c|}{$\operatorname{Re}\left(\omega_{2}\right)$} & \multicolumn{2}{c|}{$\operatorname{Im}\left(\omega_{2}\right)$} \\
& current & $\exp [2]$ & current & $\exp [2]$ & current & $\exp [2]$ & current & $\exp [2]$ \\
\hline 0 & 3.5160 & 3.519 & 0 & 0 & 22.034 & 22.034 & 0 & 0 \\
\hline 2 & 3.552 & 3.558 & 1.889 & 1.888 & 20.993 & 20.996 & 1.688 & 1.691 \\
\hline 4 & 4.274 & 4.277 & 4.784 & 4.811 & 17.441 & 17.445 & 2.371 & 2.375 \\
\hline 6 & 3.084 & 3.090 & 12.187 & 12.191 & 13.424 & 13.431 & -1.454 & -1.457 \\
\hline 8 & 0 & 0 & 12.447 & 12.449 & 12.740 & 12.745 & -5.548 & -5.551 \\
\hline 10 & 0 & 0 & 14.834 & 14.838 & 12.664 & 12.668 & -8.870 & -8.874 \\
\hline
\end{tabular}

shapes where obtained and compared to the current results and found to be in excellent agreement.

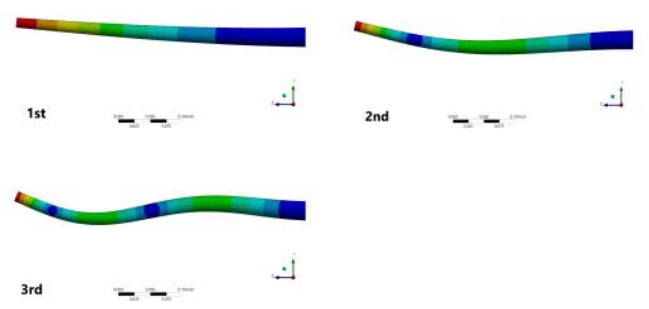

Fig. 2 The first three mode shapes for $b / a=1.42$, $B=0.223$.

The argand diagram for complex real and imaginary part of the first fundamental frequency for different b/a ratios are presented in Fig. 2. The effect of b/a ratios on the behavior of such complex frequency is complicated in which it has nonzero pure real part then becomes real and imaginary parts at certain flow velocity, namely, $U=4.4,1.6$ and 1 for $\mathrm{b} / \mathrm{a}=1$, 1.8 and 2, respectively. This indicates that in case of pure real part, the system goes only in oscillating pattern, while when it moves to have real and imaginary parts, they system will have the damping oscillating effect which may possess instability for negative imaginary part as clearly shown in literature [2] for second natural frequencies at certain configuration. Hence, the main challenge for the design requirement for the cantilevered pipe conveying fluid is to set up the parameters in an attempt to avoid unstable regions. Therefore, it would be more convenient to present these complex parts of fundamental frequencies separately in an attempt to understand the effect of physical parameters. Fig. 3 shows the effect of increasing flow velocity on the imaginary part of the first natural frequencies for different cross section ratio, $b / a$, at mass fraction $B=0.200$. in this figure, the imaginary part for, $b / a=1.0$, remains zero until the fluid reaches a velocity $U=4.5$ where the imaginary part becomes nonzero indicating oscillating damping behavior, meanwhile this behavior starts at smaller flow velocities for $b / a=1.8$ and 2.0. It can be stated that this behavior is dependent on the value of mass fraction $B$ and cross section ratio $b / a$ as it will be shown later. The behavior of the real part of the first natural frequency is dependent on each value of $U$ as shown in Fig. 4. The system oscillation is fading at $U=1.8$ for $b / a=2.0$ in an earlier stage compared to $b / a=1.0(U=10.5)$. The effect of cross section diameters $b / a$ on the real (dashed) and imaginary (solid) parts of the first two fundamental frequencies, at velocity $U=4$, and mass fraction, $B=0.2$, is shown in Fig. 5. In general, there is a reciprocal behavior between real and imaginary parts when varying the cross section ratio.

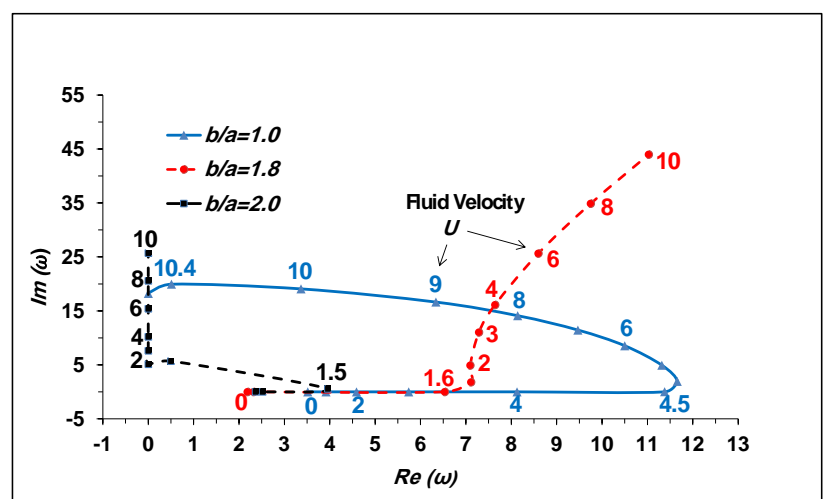

Fig. 3 The complex diagram for first natural frequency for different b/a ratios with $B=0.2$. 


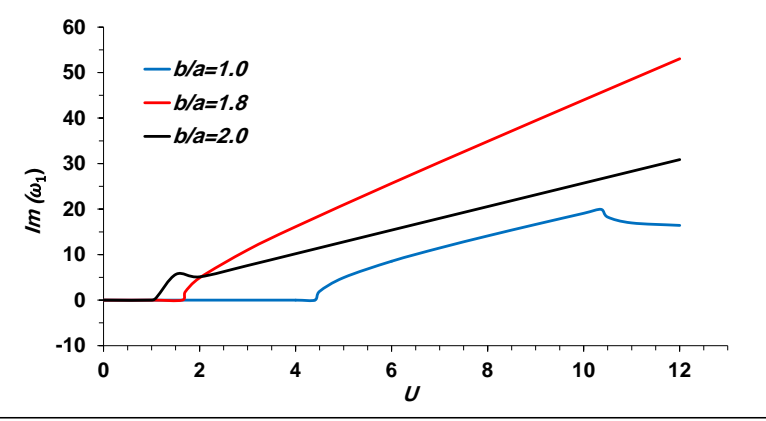

Fig. 4 The impact of flow velocities toward the imaginary part of the first frequency for different $b / a$ ratios with $B=0.2$.

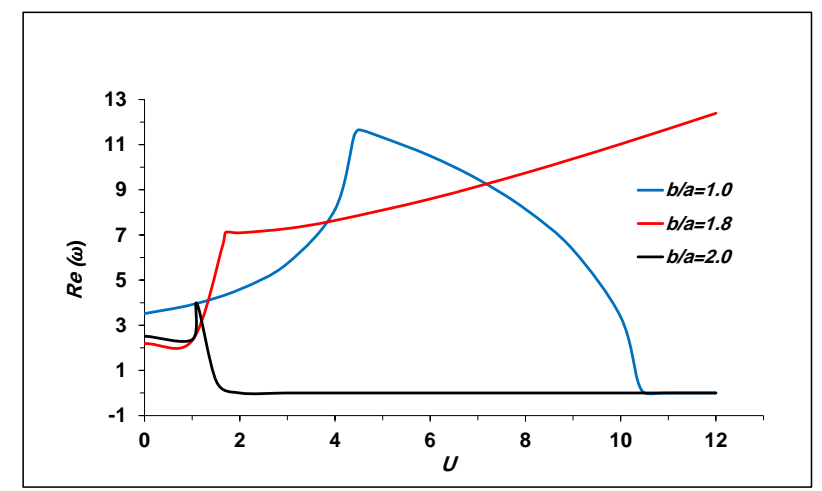

Fig. 5 The impact of flow velocities toward the real part of the first frequency for different $b / a$ ratios with $B=0.2$.

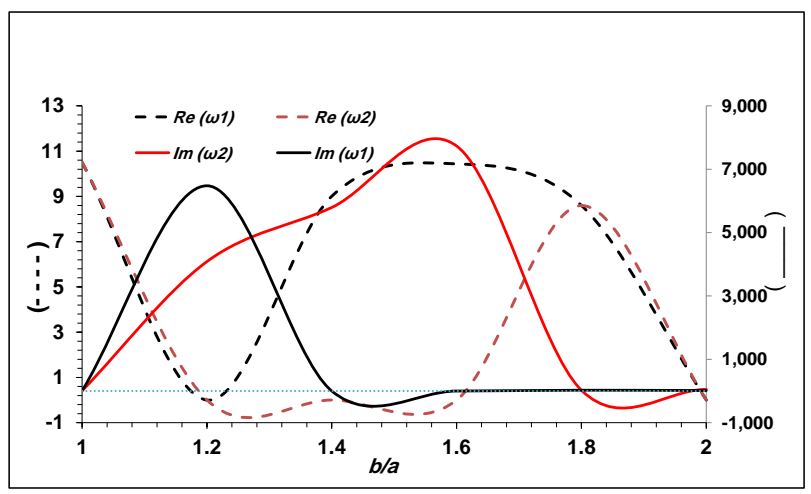

Fig. 6 The effect of b/a on the real (dashed - - -) and imaginary (solid ) parts for $\omega_{1}$ and $\omega_{2}$ with $B=0.2$.

Fig. 6 displays the complex diagram with real and imaginary of $\omega_{1}$ and $\omega_{2}$, and revealing the influence of mass fractions $B$. As gradually elevating the fluid velocity, the real part of second fundamental frequency is decreasing with nonzero imaginary parts till certain velocity. This figure shows that the imaginary part for $\omega_{2}$ (solid line) is decreasing and passing from positive to negative part flow velocity $U=5.9,7.8,8.9$ for $B=0.2,0.4,0.6$, respectively, leading to unstable flutter behavior. Fig. 7 shows the effect of $b / a$ on the real part of $\omega_{2}$ for different mass fractions $B$. In general, by increasing the cross section ratio, the value of the real part is decreasing as minimum at $b / a=1.5$ and noting that the effect of mass ratio is insignificant on the real part of $\omega_{2}$. Meanwhile, the effect of mass ratio is significant on the imaginary part of $\omega_{2}$ as shown in Fig. 8 which affect the damping behavior. Some other relevant studies can be found in [27] and [28].

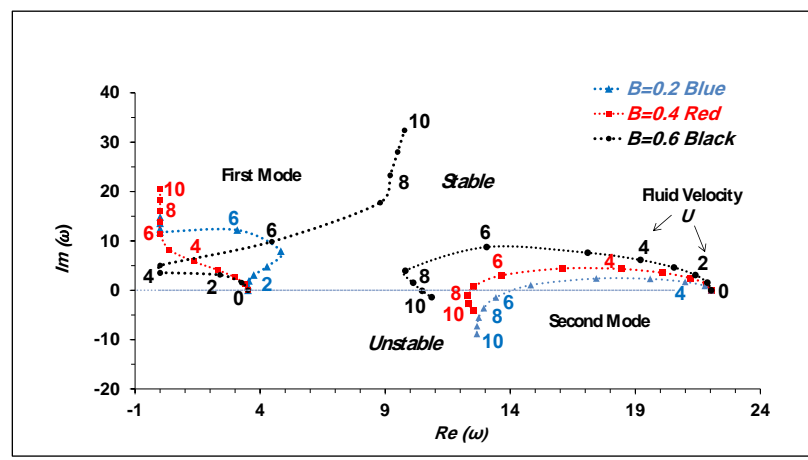

Fig. 7 The complex diagram with real and imaginary of $\omega_{1}$ and $\omega_{2}$ for different mass fractions $B$.

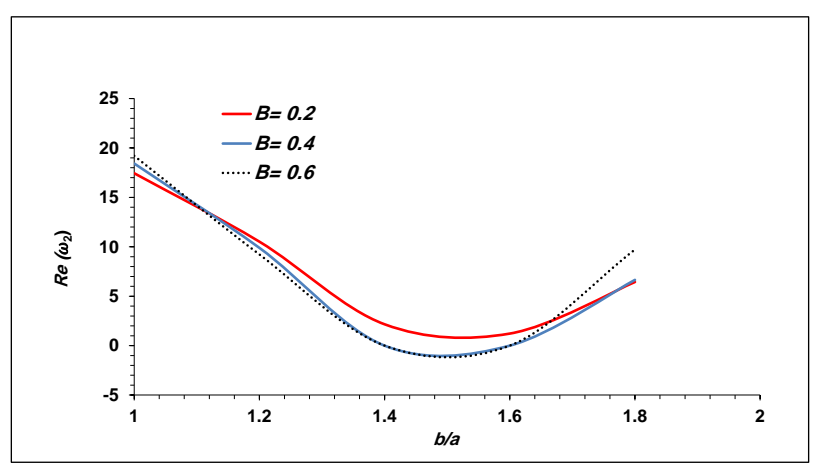

Fig. 8 The real part of second natural frequency vs $b / a$ for different mass fractions $B$. 


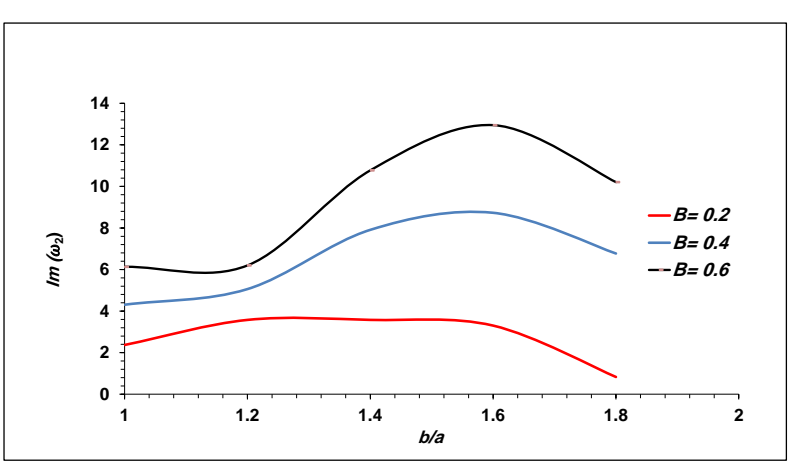

Fig. 9 The imaginary part of second natural frequency vs $b / a$ for different mass fractions $B$.

\section{Conclusiion}

The mathematical model for a pipe containing flowing flow with fixed-free ends are derived using energy methods. The effect of mass fraction $B$ and non-uniformity cross sectional ratio $\mathrm{b} / \mathrm{a}$ is investigated by analyzing its influence on the natural frequencies. It was shown that critical velocity is elevated upon raising the tapering ratio $b / a$. The results revealed that when varying the mass fraction $B$, the unstable fluttering region was altered significantly. Furthermore, it was found that the complex second fundamental frequency $\omega_{2}$ and its corresponding stability is sensitive to the values of mass fractions. In the next paper, online health monitoring for leakage detection will be presented for the current model with open edge crack using fracture mechanics and finite element method.

\section{References:}

[1] Benjamin T., Dynamics of System of Articulated Pipes Conveying Fluid-I Theory, Proc. Roy. Soc. (London), Ser. A, Vol. 261, 1961, pp. 457-486.

[2] Gregory R., Paidoussis M., Unstable Oscillation of Tubular Cantilevers Conveying Fluid. II. Experiments, Proc. Roy. Soc. (London) Ser. A, Vol. 293, 1966, pp. 528-42.

[3] Sarkar A., Paidoussis M., A Cantilever Conveying Fluid: Coherent Modes versus Beam Modes, International J. Non-Linear Mech, Vol. 39, 2004, pp. 467 - 481.

[4] Ibrahim R., Overview of mechanics of pipes conveying fluids, part I: fundamental studies, J. Press. Vessel Technol, Vol. 32, 2010, pp. 1-32.

[5] Mostafa R., Fazelzadeh S., Malihe E., Hosseini M., Flow-Induced Flutter Instability of Functionally Graded Cantilever Pipe,
International Journal of Acoustics and Vibration, Vol. 22, No. 3, 2017.

[6] Zhou K., Xiong F., Jiang N., Dai H., Yan H., Wang L, Ni Q., Vibration control of a cantilevered fluid-conveying pipe using the idea of nonlinear energy sink, Nonlinear Dynamics, Vol. 95, 2018, pp. 1-22.

[7] Liu Z., Wang L., Sun X., Nonlinear Forced Vibration of Cantilevered Pipes Conveying Fluid, Acta Mechanica Solida Sinica, Vol. 31, No. 1, 2018, pp. 32-50.

[8] Liang X., Zhaa X., Jiang X., Wang L., Leng J., Caoa Z., Semi-analytical solution for dynamic behavior of a fluid-conveying pipe with different boundary conditions, Ocean Engineering, Vol. 163, 2018, pp. 183-190.

[9] Giniatoulline A., Mathematical Modeling of the Rotating Stratified Fluid in a Vicinity of the bottom of the Ocean, International Journal of Mechanics, Vol. 13, 2019, pp. 149-155.

[10] Matteo D. L., Vedova D., Alimhillaj P., Novel Fluid Dynamic Nonlinear Numerical Models of Servovalves for Aerospace, International Journal of Mechanics, Vol. 13, 2019, pp. 3951.

[11] Liu, Z., Zhou, K., Wang, L., Jiang, T., Dai, H. (2019). 'Dynamical stability of cantilevered pipe conveying fluid in the presence of linear dynamic vibration absorber', Journal of Computational Applied Mechanics, 50(1), pp. 182-190.

[12] Zhou K., Niab O., Daia H.,Wang L., Nonlinear forced vibrations of supported pipe conveying fluid subjected to an axial base excitation, Journal of Sound and Vibration, Vol. 471, 2020, pp. 115189.

[13] Sazesh S., Shams S., Vibration analysis of cantilever pipe conveying fluid under distributed random excitation, Journal of Fluids and Structures, Vol. 87, 2019, pp. 84101.

[14] Guo Q., Liu Y., Chen B., Zhao Y., An efficient stochastic natural frequency analysis method for axially varying functionally graded material pipe conveying fluid, European Journal of Mechanics - A/Solids, Vol. 86, 2021, pp. 104155.

[15] Paidoussis M., Dynamics of tubular cantilevers conveying fluid, The Journal of Technical Engineering Science, Vol. 12, 1970, pp. 85103. 
[16] Paidoussis M., Issid N., Dynamic stability of pipes conveying fluid, Journal of Sound and Vibration, Vol. 33, 1974, pp. 267-294.

[17] Ariaratnam S., Namachchivaya N., Dynamic stability of pipes conveying pulsating fluid, Journal of Sound and Vibration, Vol. 107, Issue 2, 1986, pp. 215-230.

[18] Johnson1 R., Stoneking J., Carley T., Unstable oscillation of tubular cantilevered beams conveying a compressible fluid, Nuclear Engineering and Design, Vol. 96, Issue 1, 1986, pp. 33-45.

[19] Sugiyama Y., Kawagoe H., Kishi T., Nishyama S., Studies on the Stability of Pipes Conveying Fluid: (The Combined Effect of a Spring Support and a Lumped Mass), JSME International Journal, Vol. 31 Issue 1, 1988, pp. 20-26.

[20] Wang Z., Liu Y., Transverse vibration of pipe conveying fluid made of functionally graded materials using asymplectic method, Nuclear Engineering and Design, Vol. 298, 2016, pp. 149-159.

[21] Pisarski D., Konowrocki R., Szmidt T., Dynamics and optimal control of an electromagnetically actuated cantilever pipe conveying fluid, Journal of Sound and Vibration, Vol. 432, 2018, pp. 420-436.

[22] Bai Y., Xie W., Gao X., Xu W., Dynamic analysis of a cantilevered pipe conveying fluid with density variation, Journal of Fluids and Structures, Vol. 81, 2018, pp. 638-655.

[23] Wang X., Yang Y., Wu T., Model studies of fluid-structure interaction problems, CMES Computing Model Engineering, Vol. 119, 2019, pp. 5-34.

[24] Hannoyer M., Paidoussis M., Dynamics of slender tapered beams with internal or external axial flow 1 theory, Journal of Applied Mechanics-Transactions of the ASME, Vol. 46, Issue 1, 1979, pp. 45-51.

[25] Hannoyer M., Paidoussis M., Dynamics of slender tapered beams with internal or external axial flow 2 experiments, Journal of Applied Mechanics-Transactions of the ASME, Vol. 46, Issue 1, 1979, pp. 52-57.

[26] Gaith M., The Vibration of Tubular Beam Conveying Fluid with Variable Cross Section, Periodica Polytechnica Mechanical Engineering, Vol. 65, 2021, pp. 56-62.

[27] Gaith M., The Vibration of Simply Supported Non-Uniform Cross Sectional Pipe Conveying Fluid Resting on Viscoelastic Foundation,
WSEAS Transactions on Fluid Mechanics, Vol. 15, 2020, pp. 163-171.

[28] Oscar Bautista, Andres Matias, Jose Arcos, Pablo Escandon, Electrokinetically-driven Viscoelastic Fluid Flow in a Microchannel with Hydrodynamic Slipwalls, Pages 1-9, Engineering World, Volume 2, 2020, ISSN: 2692-5079

\section{Creative Commons Attribution License 4.0 (Attribution 4.0 International, CC BY 4.0)}

This article is published under the terms of the Creative Commons Attribution License 4.0 https://creativecommons.org/licenses/by/4.0/deed.en_US 